\title{
Dealing with Emotional Factors in Agent Based Ubiquitous Group Decision
}

\author{
Goreti Marreiros ${ }^{1}$, Carlos Ramos ${ }^{1}$, and José Neves ${ }^{2}$ \\ ${ }^{1}$ GECAD, Knowledge Engineering and Decision Support Group, \\ Institute of Engineering, Polytechnic of Porto, Porto, Portugal \\ \{goreti, csr\}@dei.isep.ipp.pt \\ ${ }^{2}$ University of Minho, Braga, Portugal \\ jneves@di. uminho.pt
}

\begin{abstract}
With the increasing globalization of economy and consequent increasing in the inter and intra organizational competitiveness, the role of groups in organizations and businesses achieve greater significance. The work, as well as the responsibility involved to reach a decision, is distributed among group members, which may be distributed geographically and may cooperate in an asynchronous way. This paper shortly presents the WebMeeting prototype, which is a group decision support system that supports ubiquitous group decision meetings. It is also discussed the influence of emotional factors in group decision making and it is proposed a multi-agent model to simulate ubiquitous group decision making processes, where argumentation and emotional capabilities are considered.
\end{abstract}

\section{Introduction}

The problem of group decision-making has gained great relevance in the scope of Decision Support Systems, which were initially designed as individual tools. Quickly those tools have demonstrated to be limited, in the sense that in today's organizations several persons, entities or agents are involved in most of the decision processes. In that way decision problems are considered from different points of view, with different opinions about the importance of the decision criteria (for instance, in the purchase of a car we will be able to consider criteria like price, technical characteristics, design or manufacturer).

The present business environment is characterized by the use of groups, which work in distributed environments and have to deal with uncertainty, ambiguous problem definitions, and rapidly changing information.

In order to support group work, numerous commercial and non commercial Group Decision Support Systems (GDSS) were developed in the last years (GroupSystems software; WebMeeting [16]; HERMES [10]; VisionQuest software). Despite the quality of these systems, they present some limitations. In our recent work we are proposing some new ideas to deal with GDSS [14], namely: the use of Multi-Agent Systems to model group participants; and the inclusion of argumentation and emotional aspects in the group decision making process. 
The work described in this paper is included in ArgEmotionAgents project (POSI / EIA / 56259 / 2004 - Argumentative Agents with Emotional Behaviour Modelling for Participants' Support in Group Decision-Making Meetings), which is a project supported by FCT (Science \& Technology Foundation - Portugal) envisaging the use of Multi-Agent Systems approach for simulating Group Decision-Making processes, where Argumentation and Emotion components are specially important.

This paper is organized as follows. Section 2 provides a general approach to group decision making, in particular to ubiquitous group decision making. Yet in this section it is presented the WebMeeting prototype (which is a group decision support system that supports ubiquitous group decision meetings) and is discussed the role of emotion in group decision meetings. In section 3 it is presented the OCC model of emotion proposed by Ortony, Clore and Collins [20]. A model to support agent based ubiquitous group decision making is proposed in section 4, this model has several components, we will focus in the emotional component. Finally section 5 presents conclusions and gives some perspectives and ideas for future work.

\section{Group Decision}

The term Group Decision Support System (GDSS) [7][12] emerged effectively in the beginning of the eighty-decade. According to Huber [8] a GDSS consists of a set of software, hardware, languages components and procedures that support a group of people engaged in a decision related meeting. A more recent definition from Nunamaker and colleagues [18] says that GDSSs are interactive computer-based environment which support concerted and coordinated team effort towards completion of joint tasks.

Generically we may say that GDSS aims to reduce the loss associated to group work (e.g. time consuming, high costs, improper use of group dynamics, etc.) and to maintain or improve the gains (e.g. groups are better to understand problems and in flaw detection, participants' different knowledge and processing skills allow results that could not be achieved individually). The use of GDSS allows groups to integrate the knowledge of all members into better decision making.

Jonathan Grudin [6] classifies the digital technology to support the group interaction in three phases: pre-ubiquitous, the proto-ubiquitous and the ubiquitous. In the pre-ubiquitous phase, that begin in the 70's, were supported face-to-face meetings. In the proto-ubiquitous phase distributed meetings were supported, this phase begun approximately at 1990. The ubiquitous phase is now getting under way and support meetings distributed in time and space. This classification is similar to DeSancits and Gallupe [3] classification to GDSS, although in this last one it is considered another type of support, the Local Decision Network that is a type of support where group members meet at the same place but at different time.

Our interest is in ubiquitous group support.

\subsection{Ubiquitous Group Decision Making}

There are many areas where ubiquitous group decision making makes sense. One of the most cited areas in literature is Healthcare since patient's treatment involves various specialists, like doctors, nurses, laboratory assistants, radiologist, etc. These 
specialists could be distributed across departments, hospitals or even in different countries. The HERMES system, a web-based GDSS was tested inside this context [10]. Many of the decisions we take every day will take a new dimension if we consider that they will be resolved by a group of individuals, for instance: choice of a place to make vacations, buy a car, hire an employee and choice of a place to build a new airport. If the group members are dispersed in time and space, the need of coordination, informal and formal communication, and information share support will increase significantly. There are already some examples of GDSS that support ubiquitous decision (GroupSystems software; WebMeeting [16]; HERMES [10]; VisionQuest software).

\subsection{Emotion in Group Decision}

Common sense usually tell us that a great deal of emotion can harm decision making process but, on the other hand, Rosalind Picard for instance, claims that too little emotion can impair decision making as well [21]. It seams that, in decision making processes, emotion is needed in a balanced way.

In psychological literature several examples could be found on how emotions and moods affects the individual decision making process:

- Individuals are more predisposed to recall past memories that are congruent with their present feelings.

- Positive mood trend to promote risk aversion behaviour, while negative mood promote a risk taking behaviour.

- Positive moods tend to be associated with heuristics information strategy processing, while negative moods are more related to systematic processing.

Emotion will influence the individual decisions of the group members, but during a group decision making, group members may be also influenced by the displayed emotions of other members.

The process of emotional contagion could be analysed based on the emotions that a group member is feeling or based on the group members mood [17].

A more detailed review of the influence of emotion in group decision making can be found in [13].

\subsection{WebMeeting Prototype}

WebMeeting is a GDSS that supports distributed and asynchronous meetings through the Internet (ubiquitous meetings) [16]. The WebMeeting system is focused on multicriteria problems, where there are several alternatives that are evaluated by various decision criteria. Moreover the system is intended to provide support for the activities associated with the whole meeting life cycle, from the pre-meeting phase to the postmeeting phase.

The system aims at supporting the activities of two distinct types of users: ordinary group "members" and the "facilitator". The users of WebMeeting can access the system from anywhere through a PC and an Internet connection.

The WebMeeting system is composed by the following modules: Setup, Management, Argumentation, Multi-criteria, Voting and Database. The Setup module will be 
operated by a facilitator during the pre-meeting phase. The Multi-Criteria module is used: by the facilitator during the pre-meeting phase to configure the multi-criteria decision problem; and by the participants during the meeting in order to establish individual preferences. The argumentation module is based on the IBIS (Issue Based Information System) argumentation model [18] and implements an argumentation forum where group members could argue in favor or against alternatives. The Voting module is responsible for the emission of "vote bulletins", and for the publication of results (intermediate and final). In figure 1 it is possible to see a screen of an argumentation forum of a very simple group decision (acquisition of a laptop).

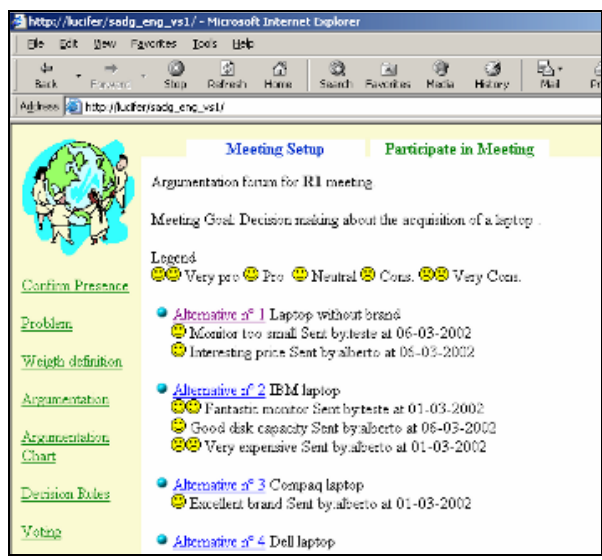

Fig. 1. Argumentation forum

An interesting and somehow natural expansion of the WebMeeting system might involve the addition of a simulation system where it should be possible to simulate the participants of an ubiquitous group decision meeting through emotional autonomous agents. Bellow it will be described some of the approaches that can be found in literature, that use agents and in particular multi-agent systems in group decision support systems. Section 4 will present our model of an agent based support to ubiquitous decision that handles emotional aspects.

\subsection{Agents in Group Decision Support Systems}

In literature there are already descriptions of agent based GDSS, some of them will be described afterwards.

Ito and Shintani [9] propose an architecture for an agent based GDSS where, it is associated an agent to each member (human) of the decision meeting. The key idea of this system is the persuasion mechanism between agents. The persuasion in this system is already done in pairs, for instance, agent A tries to convince agent B about the choice of alternative $X$, if agent $A$ succeed then they will form a group and together will start a new persuasion cycle and try to convince another agent about the choice of alternative $\mathrm{X}$. 
Kudenko and colleagues [11] propose a system named MIAU whose aim is to support a group of users in the decision of acquiring a good from an electronic catalogue. The catalogue items are characterized by a set of criteria (if the item of the catalogue is a car the criteria could be: price, technical characteristics, design or manufacturer, capacity of charge). MIAU intends to obtain a compromise solution that can be acceptable for all group members and for that it acquires the preference models of each user through interface agents. After this phase a mediator agent combine all the agents and try to identify negotiable aspects and to suggest what seems to be a compromise solution. The users can accept or reject the proposed solution, and that may imply updates in the individual preference models. This process is repeated until a consensual solution is found.

Hermes [10] is a web-based GDSS that supports argumentative discourses between group members. The role of agents in this system is, for instance, to provide mechanisms to validate arguments consistency as well as to weight them. Agents in Hermes are also responsible for processes related with information search, for instance recovering information from previous discussions.

\section{OCC Model}

As we have seen before, the emotional state of an individual affects its decisions and influence the emotional state of others member of the group, through the process of emotional contagion that will be discussed in section 4 . As we intend to simulate group decision making through autonomous agents, it is important that those agents have some emotional characteristics, in order to approximate the simulation to the reality.

The OCC model [20] proposes that emotions are the results of three types of subjective appraisals:

1. The appraisal of the pleasantness of events with respect to the agent's goals.

2. The appraisal of the approval of the actions of the agent or another agent with respect to a set of standards for behaviour.

3. The appraisal of the liking of objects with respect to the attitudes of the agent.

Generically in the OCC model emotions are seen as valenced reactions to three different type of stimulus [20]: objects; consequence of events and action of agents. These are the three major branches of emotion types. In the branch objects we have the emotions love and hate. In the branch consequences of events we have the emotions: happy-for, gloating, pity, resentment, satisfaction, hope, fear, fears-confirmed, relief, disappointment, joy and distress. In the branch actions of agents we have the emotions: pride, shame, admiration and reproach. The model considers yet 4 compound emotions, because they are consequence of events and agents actions, which are: gratification, remorse, gratitude and anger.

The original OCC model, described above, with his 22 different types of emotions is probably, for our propose, to much fine grained. A simplified version of this theory was presented in 2003 by Ortony [19], where he considered only two different categories of emotional reactions: positive and negative. As in the original model, emotions are the results of three types of subjective appraisals (goal-based, standard-based and taste-based). In table 1 it is possible to visualize the OCC model reviewed in 2003, after the collapse of some of the original categories. 
Table 1. Five specializations of generalized good and bad feelings (collapsed from [19])

\begin{tabular}{|c|c|c|}
\hline & Positive Reactions & Negative Reactions \\
\hline Undifferentiated & $\begin{array}{l}\text {...because something good happened } \\
\text { (joy) }\end{array}$ & $\begin{array}{l}\text {...because something bad happened } \\
\text { (distress) }\end{array}$ \\
\hline \multirow{2}{*}{ Goal-based } & $\begin{array}{l}\text {...about the possibility of something } \\
\text { good happening (hope) }\end{array}$ & $\begin{array}{l}\text {...about the possibility of something bad } \\
\text { happening (fear) }\end{array}$ \\
\hline & $\begin{array}{l}\text {... because a feared bad thing didn't } \\
\text { happen (relief) }\end{array}$ & $\begin{array}{l}\ldots \text { because a hoped-for good thing didn't } \\
\text { happen (disappointment) }\end{array}$ \\
\hline \multirow{2}{*}{ Standard-based } & $\begin{array}{l}\ldots \text { about a self-initiated praisewor- } \\
\text { thy act (pride) }\end{array}$ & $\begin{array}{l}\text {.. about a self-initiated blameworthy act } \\
\text { (remorse) }\end{array}$ \\
\hline & $\begin{array}{l}\ldots \text { about an other-initiated praise- } \\
\text { worthy act (gratitude) }\end{array}$ & $\begin{array}{l}\text {...about an other-initiated blameworthy } \\
\text { act (anger) }\end{array}$ \\
\hline Taste-based & $\begin{array}{l}\ldots \text { because one finds someone/thing } \\
\text { appealing or attractive (like) }\end{array}$ & $\begin{array}{l}\ldots \text { because one finds someone/thing } \\
\text { unappealing or unattractive (dislike) }\end{array}$ \\
\hline
\end{tabular}

The OCC model was several times used to model the implementation of emotional agents, and afterwards we will referrer to some of the implementations that use it.

Bates [2] developed the OZ project in which real-time, interactive, self-animating agents were situated in simulated micro-worlds. These agents, who were based on the principles of traditional character animation, were equipped with emotions to make them believable. The module that implements emotions in the OZ project is the EM module that is based in a simplified version of the OCC model (only some emotions of the model were implemented).

Elliot [4] developed the Affective Reasoner, a multi-agent simulation model based on the OCC emotions model, where agents have the capacity to produce twenty four emotion types and express more than 1200 facial expressions. Each agent has a representation of itself and a representation of the concerns of other agents which allow them to explain the emotional episodes of others. During the simulation, agents judge events according to their attractiveness and status (unconfirmed, confirmed, and disconfirmed).

Adamatti and Bazzan in [1] describe Afrodite, a framework to simulate agents with emotions that is based on the OCC model. With this simulation framework it is possible to configure different scenarios.

El-Nasr [5] proposes the FLAME model that is a computational implementation of emotions that uses fuzzy logic and is based in a combination of the OCC model and the Roseman emotion model [22].

Despite several implementations of the OCC model, it is not exempt of critics, probably the more cited are: the fact that OCC model does not retain memory of past emotions (interactions) and the impossibility to model an emotion mixture.

\section{The Proposed Model}

As we referred in the beginning of this paper our aim is to present a multi-agent model to simulate ubiquitous group decision making considering emotional factors. In our opinion the use of Multi-Agent Systems seems to be quite suitable to simulate the behaviour of groups of people working together and, in particular, to ubiquitous group decision making modelling, because it allows [15]: 
- Individual modelling - each participant of the group decision making can be represented by an agent that will interact with other agents. Agents can be modelled with social and emotional characteristics in order to become more realistic.

- Flexibility - with this approach it is easy to incorporate or remove entities.

- Data distribution - frequently, in group decision making, participants are geographically distributed.

In ours previous work we identified the main agents involved in a simulation of a group decision meeting [14] and they are: Participant Agents; Facilitator Agent; Register Agent; Voting Agent and Information Agent.

In the remain text of this section we will first present the architecture of participants agents, because they represent the main role in group decision making and then we will detail one of the components of this architecture, the Emotional module.

\subsection{Participant Agent Architecture}

In figure 2 it is represented the architecture of participant agents. This architecture contains three main layers: the knowledge layer, the reasoning layer and the communication layer.

In the knowledge layer the agent has information about the environment where it is situated, about the profile of the other participants agents that compose the simulation group, and regarding its own preferences and goals (its own profile). The information in the knowledge layer is dotted of uncertainty and will be accurate along the time through interactions done by the agent.

The communication layer will be responsible for the communication with other agents and by the interface with the user of the group decision making simulator.

The reasoning layer contains three major modules:

- the argumentative system - that will be responsible by the arguments generation;

- the decision making module - that will choose the preferred alternative;

- the emotional system - that will generate emotions and moods, affecting the choice of the arguments to send to the others participants, the evaluation of the received arguments and the final decision.

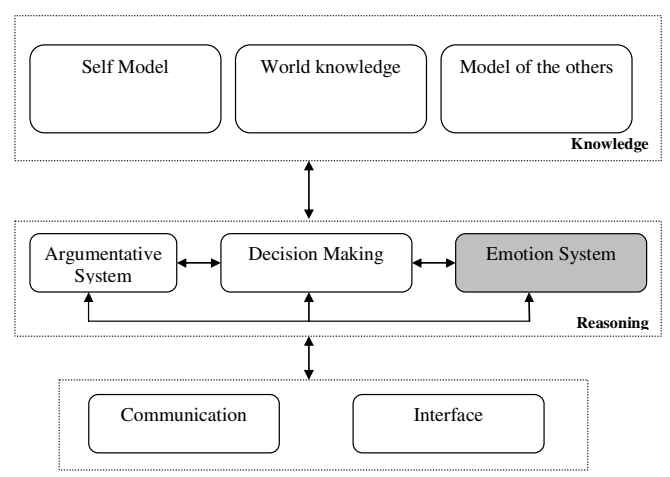

Fig. 2. Participant Agent Architecture 


\subsection{Emotional Module}

The emotions that will be simulated in our system are those identified in the reviewed version of the OCC model: joy, hope, relief, pride, gratitude, like, distress, fear, disappointment remorse, anger and dislike.

An emotion in our system is characterized by the proprieties identified in table 2 .

Table 2. Emotion property

\begin{tabular}{|l|l|}
\hline Type & Emotion type \\
\hline Valence & Positive or negative \\
\hline Agent_Id & Identification of the agent \\
\hline Time & Moment in time when emotion was initiated \\
\hline Origin_Id & Identification of the agent or event that origin the emotion \\
\hline Intensity & Emotion intensity \\
\hline
\end{tabular}

In figure 3 it is possible to visualize the main components of the emotional system.

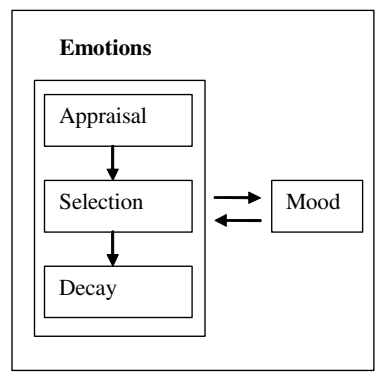

Fig. 3. Emotional Module

The emotional module is composed by three main components: the appraisal based on OCC model, where the intensities of potential emotions are calculated; the selection - each emotion has a threshold activation, that can be influenced by the agent mood, this component selects the dominant emotion; and decay - emotions have a short duration, but they do not go away instantaneously, they have a period of decay.

The agent mood is calculated based on the emotions agents felt in the past and in the moods of the remaining participants. In our approach only the process of mood contagion is being considered, we do handle the process of emotions contagion. We consider only three stages for mood: positive, negative and neutral.

In group decision simulation the participant agents will exchange arguments in order to achieve a consensual solution, the selection of arguments to be sent and the evaluation of received arguments will take into account the agent internal emotional state, the moods of other agents, as well as other characteristics that compose the agents profile: debts of gratitude, agents in which the participant agent trust, agents that participant agent think that consider him as credible, friendship agents and enemy agents. 
Although our model is based on the OCC model we think that with the inclusion of mood we can surpass one of the major critics that usually is pointed to this model, the fact that OCC model does not handle treatment of past interactions, past emotions.

\section{Conclusion}

More and more organizational decisions are taken by groups of people distributed in time and space. It is also accepted that the emotional state of an individual affects its decision and when he is taking part of a group decision he will influence both the emotional state of others members and group decisions.

In this paper it was briefly presented WebMeeting a ubiquitous group decision support system, but it main goal was the presentation of an agent based simulation model to group decision. The presented model incorporate the agents emotions and mood in the decision making process. The agent emotions and mood affect the selection of arguments to send to others agents, as well as, the evaluation of the received arguments. Agents individual emotions and mood are affected by the process of mood contagion.

Future work includes the implementation, validation and consequent refinement of the model. The inclusion of this model as component of WebMeeting is also being considered. In that case a participant in a real ubiquitous group decision meeting, supported by WebMeeting, will use this model for instance to simulate the other participants and to preview its behaviour.

\section{References}

1. Adamatti, D. and Bazzan, A.: AFRODITE - A Framework for Simulation of Agents with Emotions. ABS 2003 - Agent Based Simulation 2003. Montpelier, France, 28-30 April, (2003)

2. Bates, J.: The role of emotion in believable agents. Communications of the ACM, Special Issue on Agents, July (1994)

3. DeSanctis, G. and Gallupe, R. B.: Group Decision Support Systems - A New Frontier. Database Vol. 16 No. 1 (1985) 3-10

4. Elliot, C.: The Affective Reasoner A process model of emotions in a multi-agent systems. PhD dissertation. Northwestern University, USA, (1992)

5. El-Nasr, M.; Yen, J.; Ioerger, T.R.: FLAME -Fuzzy Logic Adaptive Model of Emotions. Autonomous Agents and Multi-agent systems, Vol.3 (2000) 217-257

6. Grudin, J.: Group Dynamics and Ubiquitous computing. Communications of the ACM, vol 45 No. 12 (2002)

7. Huber, G. P. : Group decision support systems as aids in the use of structured group management techniques. Proc. of second international conference on decision support systems, San Francisco, (1982) in C. W. Holsapple, A. B. Whinston, Decision support systems: a knowledge-based approach (Thomson Learning, inc, 2001)

8. Huber, G. P: Issues in the design of group decision support systems, Mis Quarteley, Vol. 3 No. 8 (1984).

9. Ito, T.; Shintani, T.: Persuasion among agents: An approach to implementing a group Decision Support System based on multi-agent negotiation. Proceedings of the $5^{\text {th }}$ International joint Conference on Artificial Intelligence (1997) 
10. Karacapilidis, N.; Papadias, D.: Computer supported argumentation and collaborative decision making: The Hermes system, Information Systems, Vol. 26 No. 4 (2001) 259-277

11. Kudenko, D.; Bauer, M.; Dengler, D.: Group decision making through mediated discussions. Proceedings of the tenth International conference on user modelling (UM'03) (2003)

12. Lewis, L.F.: Facilitator: A microcomputer decision support systems for small groups, $\mathrm{Ph}$. D. dissertation, University of Louisville, 1982. in C. W. Holsapple, A. B. Whinston, Decision support systems: a knowledge-based approach (Thomson Learning, inc, 2001).

13. Marreiros, G.; Ramos, C. and Neves, J.: Emotion and Group Decision Making in Artificial Intelligence. Proceedings of InterSymp 2005 17th International Conference on System Research, Informatics \& Cybernetics - Special Focus Symposium on Cognitive, Emotive and Ethical Aspects of Decision-Making in Humans and in AI, Baden-Baden, Germany (2005)

14. Marreiros, G.; Ramos, C. and Neves, J.: Modelling group decision meeting participants with an Agent-based approach. Selected for publication in an upcoming special issue of the International Journal of Engineering Intelligent Systems (2005)

15. Marreiros, G.; Santos, R.; Ramos, C. and Neves, J.: Agent Based Simulation for Group Formation. SCS-ESM 2005 19th European Simulation Multi-Conference, Riga, Latvia (2005) 521-526

16. Marreiros, G.; Sousa, J.P. and Ramos, C: WebMeeting - A Group Decision Support System for Multi-criteria Decision Problems. International Conference on Knowledge Engineering and Decision Support, Porto, Portugal ICKEDS04 (2004) 63-70

17. Neumann, R. and Strack, F.: Mood contagion: The automatic transfer of mood between persons; Journal of Personality and Social Psychology, Vol. 79 (2000) 211-223

18. Nunamaker, J.F. et al.: Lessons from a dozen years of group support systems research: A discussion of lab and field findings. Journal of Management Information Systems, Vol. 13 No. 3 (1997).

19. Ortony, A.: On making believable emotional agents believable. In R. P. Trapple, P. (Ed.), Emotions in humans and artefacts. Cambridge: MIT Press (2003)

20. Ortony, A.; Clore, GL; Collins, A.: The cognitive structure of emotions. Cambridge: Cambridge University Press (1988)

21. Picard, R. : Affective Computing; MIT Press, Cambridge, MA (1997)

22. Roseman, I.; Spindel, M.; Jose, P.: Appraisals of emotion-eliciting events: Testing a theory of discrete emotions. Journal of Personality and Social Psychology, Vol. 59, (1990) 